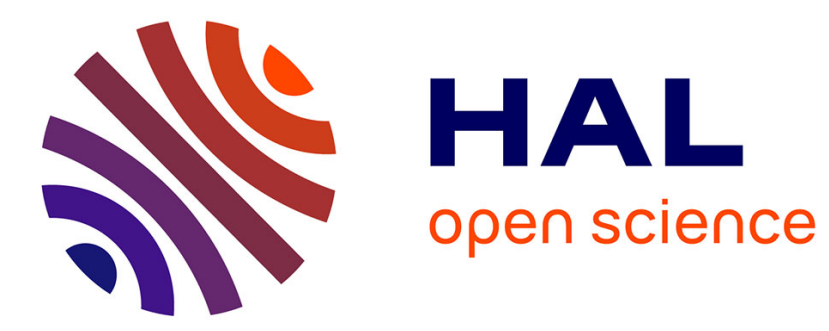

\title{
Hydrolyse du lactose contenu dans l'ultrafiltrat de lait ou de lactosérum en réacteur enzymatique à membrane
}

L. Roger, J.L. Thapon, J. L. Maubois, G. Brule

\section{To cite this version:}

L. Roger, J.L. Thapon, J. L. Maubois, G. Brule. Hydrolyse du lactose contenu dans l'ultrafiltrat de lait ou de lactosérum en réacteur enzymatique à membrane. Le Lait, 1976, 56 (551_552), pp.56-75. hal-00928715

\section{HAL Id: hal-00928715 https://hal.science/hal-00928715}

Submitted on 1 Jan 1976

HAL is a multi-disciplinary open access archive for the deposit and dissemination of scientific research documents, whether they are published or not. The documents may come from teaching and research institutions in France or abroad, or from public or private research centers.
L'archive ouverte pluridisciplinaire HAL, est destinée au dépôt et à la diffusion de documents scientifiques de niveau recherche, publiés ou non, émanant des établissements d'enseignement et de recherche français ou étrangers, des laboratoires publics ou privés. 


\title{
Hydrolyse du lactose \\ contenu dans l'ultrafiltrat de lait ou de lactosérum en réacteur enzymatique à membrane*
}

\author{
par \\ L. ROGER, J. L. THAPON, J. L. MAUBOIS \\ et G. BRULE \\ Laboratoire de Recherches de Technologie Laitière, I.N.R.A. \\ 65, rue de St-Brieuc - 35042 Rennes Cedex
}

Le lactose est assimilé par l'organisme, essentiellement au niveau de l'intestin grêle, une fois effectuée l'hydrolyse de sa molécule en glucose et en galactose par une enzyme de la muqueuse iléale, la $\beta$ galactosidase appelée plus communément lactase.

La sécrétion de cette enzyme, maximale au moment de la naissance, décroît rapidement jusqu'à devenir nulle s'il y a arrêt de la consommation de lait. Selon Kretchmer (1971), 75 p. 100 de la population adulte mondiale seraient déficients en lactase. L'ingestion de lactose, en cas d'activité lactasique insuffisante provoque des troubles digestifs (diarrhées, vomissements, etc.), le lactose étant alors métabolisé par la flore bactérienne du gros intestin ; dans quelques rares cas d'intolérance aiguë, il peut même se produire une altération irréversible du système nerveux central (Holzel et al., 1962).

Le lait et ses dérivés à lactose partiellement ou totalement hydrolysé présentent donc un intérêt nutritionnel évident (Olling, 1972 ; De Waard, 1975) pour :

- les nourrissons alactasiques ou déficients en lactase ;

- les vieillards, pour qui le lait représente une source particulièrement intéressante de calcium et de protéines, mais qui n'en consomment que peu ou pas en raison des troubles digestifs entraînés par son ingestion, troubles digestifs qu'on s'accorde à attribuer à la diminution du caractère inductible de la sécrétion de lactase ;

- les enfants et les adolescents de certaines ethnies chez qui le caractère adaptatif de la sécrétion de lactase n'existerait génétiquement pas.

\footnotetext{
* Ce travail a bénéficié du concours financier de la D.G.R.S.T. (contrat n 7471047).
} 
Par ailleurs, outre leur valeur nutritionnelle, les produits dérivés du lait à lactose hydrolysé présentent un intérêt sur les plans suivants :

- technologique : simplification de la fabrication des laits concentrés sucrés ou non en évitant la formation de gros cristaux de lactose (Hyde et Rothwell, 1973 ; Webb et al., 1974) ;

- économique : création de nouveaux produits ou de produits de substitution utilisables dans d'autres industries agro-alimentaires ce qui peut conduire à une valorisation accrue ou/et à de nouveaux débouchés pour l'industrie laitière.

Des enzymes d'origines diverses, lactases d'origine microbienne (Saccharomyces lactis; Escherichia coli) ou d'origine fongique (Aspergillus niger) ont été proposées pour l'hydrolyse du lactose du lait destiné à la consommation humaine (Kosikowski et Wierzbicki, 1973) ou à la fromagerie (Kosikowski, 1975) et du lactosérum (Giacin et al., 1974 ; Jabukowski et al., 1975). Mais l'industrialisation de cette technique est limitée par le coût élevé de l'enzyme qui doit être commercialisée sous une forme hautement purifiée puisque les produits obtenus sont destinés à l'alimentation humaine.

Aussi, pour réduire le coût d'utilisation de ces enzymes, diverses techniques d'immobilisation ont été proposées (Olson et Cooney, 1974 ; Zaborsky, 1974). L'enzyme une fois fixée sur un support solide (billes de verre, particules ou membrane de collagène, barreau aimanté recouvert de polytétrafluoroéthylène, etc.) est mis en contact avec le produit laitier à hydrolyser. Les lactases ainsi fixées, présentent en général une meilleure stabilité aux traitements physiques (température, agitation), mais leur activité demeure inférieure à celles des enzymes libres : une partie active de la molécule est en effet masquée par la fixation et les conditions optimales d'activité sont modifiées. Par ailleurs, avec de tels réacteurs à enzyme fixée, l'emploi des agents détergents et désinfectants habituellement utilisés pour le nettoyage et l'aseptisation dans l'Industrie Alimentaire est à proscrire car ces agents risquent de dénaturer irréversiblement l'enzyme. La réutilisation de ces réacteurs peut donc conduire à une contamination inadmissible des lots de lait soumis à l'hydrolyse.

Nous avons pensé que la technologie dite du réacteur enzymatique à membrane (Davis, 1974 ; Vorsilak et al., 1975) permettrait de pallier ces inconvénients. En effet, l'enzyme maintenue en solution au droit de la membrane conserve les caractéristiques de l'enzyme libre ; la solution de lactase peut être concentrée et récupérée en quasi-totalité à la fin de chaque opération. Le nettoyage et la désinfection des installations d'hydrolyse peuvent donc s'effectuer dans des conditions habituelles.

L'utilisation de tels réacteur; pour l'hydrolyse du lactose contenu dans le lait et dans ses dérivés nécessite une séparation préalable des protéines et de la phase aqueuse de ces produits. Une telle 
séparation est obtenue par la mise en contact du lait (Maubois et Mocquot, 1971) ou du lactosérum (Fallick, 1969) avec une membrane semi-perméable.

Au cours de ce travail, nous nous sommes attachés, dans une première étape, à étudier les conditions d'hydrolyse du lactose contenu dans la phase aqueuse ou ultrafiltrat obtenu lors du traitement du lait et du lactosérum par ultrafiltration sur membrane. Dans une seconde étape, nous avons tenté de définir les paramètres permettant de réaliser cette hydrolyse en continu dans un réacteur à membrane. Enfin, nous avons étudié la combinaison des opérations d'ultrafiltration du lait et d'hydrolyse de l'ultrafiltrat, combinaison dont l'extrapolation en tant que méthode industrielle de production de lait à lactose hydrolysé est envisageable.

\section{MATERIEL ET METHODES}

\section{I. - Lactase}

L'enzyme utilisée était de la lactase purifiée (40000 unités $\mathrm{ONPG}^{*} / \mathrm{g}$ ) obtenue à partir de Saccharomyces lactis par GistBrocades, Delft - Hollande.

\section{II. - Ultrafiltrats}

Les ultrafiltrats étaient obtenus par ultrafiltration à froid de lait pasteurisé H.T.S.T. de grand mélange ( $\mathrm{pH} 6,6)$, écrémé ou non, ou de lactosérum déminéralisé (reconstitué à partir de poudre et ajusté à $\mathrm{pH}$ 6,8 par addition de soude) à l'aide d'installations pilotes (module D.D.S. $4 \mathrm{~m}^{2}$ équipé de membranes D.D.S. 600 ou Rhône Poulenc $4 \mathrm{~m}^{2}$ équipé de membranes Iris $30-42$ ) ou industrielle (D.D.S. $216 \mathrm{~m}^{2}$, membranes D.D.S. 600 ).

Leurs compositions moyennes étaient :

\begin{tabular}{|c|c|c|c|c|c|c|c|c|c|}
\hline & \multirow{3}{*}{ 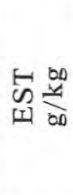 } & \multirow{3}{*}{ 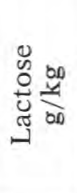 } & \multirow{3}{*}{ 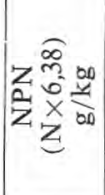 } & \multicolumn{6}{|c|}{ Sels minéraux } \\
\hline & & & & \multirow{2}{*}{$\begin{array}{l}\text { Total } \\
\mathrm{g} / \mathrm{kg}\end{array}$} & $\mathrm{Ca}$ & $P$ & $\mathrm{Mg}$ & $\mathrm{Na}$ & $\mathrm{K}$ \\
\hline & & & & & \multicolumn{5}{|c|}{ ppm } \\
\hline UF lait & 55 & 48 & 1,7 & $3-4$ & 380 & 380 & 70 & 480 & 1480 \\
\hline $\begin{array}{l}\text { UF lactosérum } \\
\text { déminéralisé }\end{array}$ & 58 & 51 & 1,8 & 1,8 & 192 & & & 640 & 160 \\
\hline
\end{tabular}

* ONPG $=$ orthonitrophénylgalactoside. 


\section{III. - Réacteur enzymatique à membrane (fig. 1)}

Sa conception était voisine de celle d'un circuit d'ultrafiltration classique (fig. 1). Nous avons utilisé un module d'ultrafiltration Romicon de type Hollow-Fiber équipé de membranes XM 50, d'une surface de $2,8 \mathrm{~m}^{2}$. Le diamètre des pores de ces membranes permettait de maintenir l'enzyme dans le circuit "rétentat ». Pendant les 20 à 30 premières minutes de fonctionnement, le perméat (liquide passant au travers de la membrane) était recyclé afin d'obtenir dans le réacteur le niveau d'hydrolyse du lactose désiré. Ce taux étant atteint, on introduisait alors de l'ultrafiltrat de lait ou de lactosérum déminéralisé dans le réacteur à un débit identique à celui du perméat.

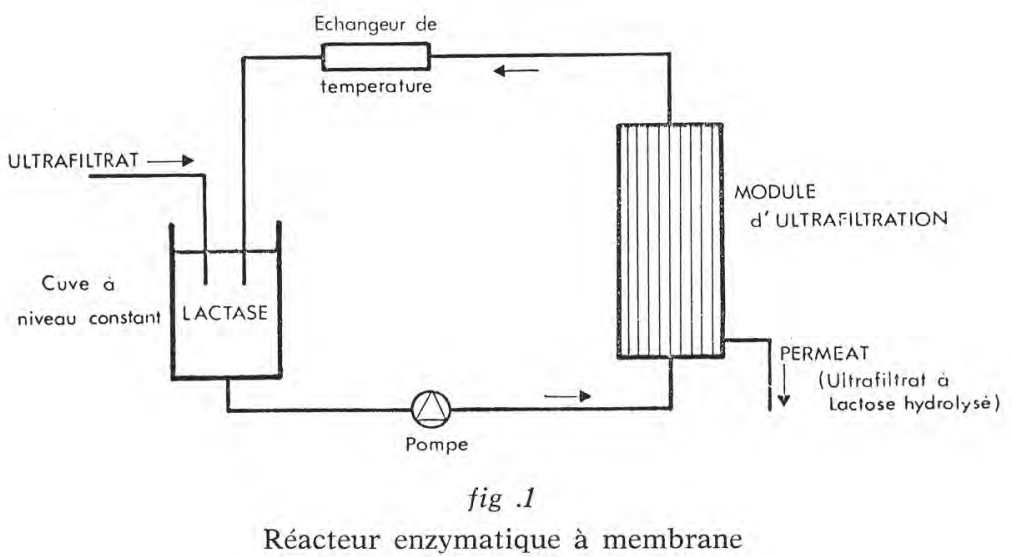

\section{IV. - Déterminations analytiques}

Les teneurs en substance sèche des échantillons de lait, de lactosérum, de rétentat et d'ultrafiltrat avant et après hydrolyse étaient déterminées par dessiccation à l'étuve à $102^{\circ} \mathrm{C}-105^{\circ} \mathrm{C}$ pendant $7 \mathrm{~h}$.

Les dosages de calcium étaient réalisés par spectrophotométrie d'absorption atomique à l'aide d'un appareil Techtron 1200 suivant le protocole de Murthy et Rhéa (1967) légèrement modifié comme suit : $1 \mathrm{ml}$ d'ultrafiltrat était dilué de 50 à 100 fois avec une solution aqueuse d'oxyde de lanthane $\left(\mathrm{La}_{2} \mathrm{O}_{3}\right)(450 \mathrm{ppm})$.

Les proportions des différents sucres présents après hydrolyse étaiént déterminées par chromatographie en phase gazeuse, à l'aide 
d'un appareil Varian série 2400 couplé à un enregistreur, selon la technique décrite par Jouanny (1972) et légèrement modifiée comme suit : 4 à $5 \mathrm{ml}$ d'échantillon étaient lyophilisés ; au résidu anhydre, on ajoutait 3 à $4 \mathrm{ml}$ de diméthylformamide, ce qui permettait de conserver l'échantillon pendant $24 \mathrm{~h}$ sans risque de réhydratation. $0,3 \mathrm{ml}$ de cette solution étaient ensuite prélevés ; on leur ajoutait $0,3 \mathrm{ml}$ d'hexaméthyldisilasane et, après mélange, $0,4 \mathrm{ml}$ de triméthylchlorosilane. Après avoir laissé reposer l'ensemble, on obtenait deux phases : les dérivés triméthylsylylés des sucres se trouvaient dans la phase surnageante : $2 \mu \mathrm{l}$ de cette dernière étaient alors injectés dans la colonne de chromatographie. Les conditions de chromatographie étaient les suivantes :

$\begin{array}{ll}\text { Température initiale } & : 160^{\circ} \mathrm{C} . \\ \text { Température finale } & : 280^{\circ} \mathrm{C} . \\ \text { Gradient de température }: & 8^{\circ} \mathrm{C} / \mathrm{mn} .\end{array}$

Nature de la colonne : Varaport imprégné d'OV $\mathrm{V}_{1}$ à 5 p. 100.

Le pourcentage de lactose hydrolysé était déterminé à partir de la surface des pics de lactose et des produits d'hydrolyse, calculée par intégrateur Varian modèle 485.

L'activité de la lactase était déterminée par un test colorimétrique en utilisant l'orthonitrophénylgalactoside (ONPG) comme substrat. $1 \mathrm{ml}$ d'une solution de lactase $(0,3$ à $0,8 \mathrm{~g}$ d'enzyme par $\mathrm{kg}$ d'ultrafiltrat), diluée 30 fois environ dans une solution tampon $\left(\mathrm{KH}_{2} \mathrm{PO}_{4}\right.$ $\mathrm{N} / 50$, pH 7) est mis en présence d'une solution d'ONPG (0,5 g d'ONPG par litre de la solution tampon phosphate monopotassique $\mathrm{N} / 50$ ajusté à $\mathrm{pH} 7$ par $\mathrm{KOH}, 50 \mathrm{p} .100$ ) pendant $10 \mathrm{mn}$, exactement à $30^{\circ} \mathrm{C}$. La réaction enzymatique est alors bloquée par addition de $2 \mathrm{ml}$ d'une solution de $\mathrm{Na}_{2} \mathrm{CO}_{3} \mathrm{~N}$. L'intensité de la coloration jaune (formation de nitrophénol) est lue à l'aide d'un spectrophotocolorimètre à $420 \mathrm{~nm}$. Les résultats de l'activité résiduelle sont exprimés en p. 100 de l'activité initiale.

\section{RESULTATS}

\section{I. - Activité de la lactase dans l'ultrafiltrat de lait}

Avant d'étudier le fonctionnement du réacteur enzymatique, il était nécessaire de connaître le comportement de l'enzyme vis-à-vis du lactose dans l'ultrafiltrat de lait.

Nous avons donc étudié l'influence de la température et du $\mathrm{pH}$ sur l'activité de la lactase et les résultats de cette étude sont résumés dans les tableaux 1 et 2 . Les conditions optimales d'activité sont voisines de celles observées dans des solutions aqueuses de lactose (Kosikowski, 1973). Le pH optimal de l'enzyme est plus bas $(6,5)$ que celui déterminé en solution de lactose $(\mathrm{pH} 7,0)$. 


\section{TABLEAU 1}

Pourcentage d'hydrolyse obtenu en fonction de la température $\mathrm{pH}$ de l'ultrafiltrat : 6,6

Concentration en lactase $: 1 \mathrm{~g} / \mathrm{l}$

\begin{tabular}{c|c|c}
\hline & \multicolumn{2}{|c}{ Pourcentage d'hydrolyse } \\
\hline Temps d'hydrolyse & $15 \mathrm{mn}$ & $30 \mathrm{mn}$ \\
\hline Température & & \\
34 & 62 & 89 \\
38 & 78 & 90 \\
42 & 61 & 65 \\
\hline
\end{tabular}

TABLEAU 2

Pourcentage d'hydrolyse obtenu en fonction du $\mathrm{pH}$

Température de l'ultrafiltrat : $35^{\circ} \mathrm{C}$

Concentration en lactase $: 1 \mathrm{~g} / 1$

\begin{tabular}{c|c|c}
\hline & \multicolumn{2}{|c}{ Pourcentage d'hydrolyse } \\
\hline Temps d'hydrolyse & $15 \mathrm{mn}$ & $30 \mathrm{mn}$ \\
\hline $\mathrm{pH}$ & & 75 \\
6 & 51 & 100 \\
6,5 & 70 & 64 \\
7 & 54 & 55 \\
7,5 & - & \\
\hline
\end{tabular}

A partir des résultats indiqués dans le tableau 3, montrant l'influence de la concentration en lactase sur la vitesse d'hydrolyse du lactose à $\mathrm{pH}$ et température optima, nous avons fixé les conditions expérimentales suivantes pour l'étude en réacteur à membrane :
Température
: $30^{\circ} \mathrm{C}-35^{\circ} \mathrm{C}$.

$\mathrm{pH}$

: $6,4-6,7$.

Concentration en lactase : $0,8 \mathrm{~g} / \mathrm{kg}$ d'ultrafiltrat. 


\section{TABLEAU 3}

Pourcentage d'hydrolyse obtenu en fonction de la concentration en lactase Température de l'ultrafiltrat : $32^{\circ} \mathrm{C}$ $\mathrm{pH}: 6,7$

\begin{tabular}{c|c|c}
\hline & \multicolumn{2}{|c}{ Pourcentage d'hydrolyse } \\
\cline { 2 - 3 } Temps d'hydrolyse & $15 \mathrm{mn}$ & $30 \mathrm{mn}$ \\
\hline $\begin{array}{c}\text { Concentration en lactase } \\
(\mathrm{g} / \mathrm{kg})\end{array}$ & & \\
0,3 & 25 & 56 \\
0,5 & 40 & 68 \\
0,8 & 65 & 85 \\
1,0 & 77 & 90 \\
\hline
\end{tabular}

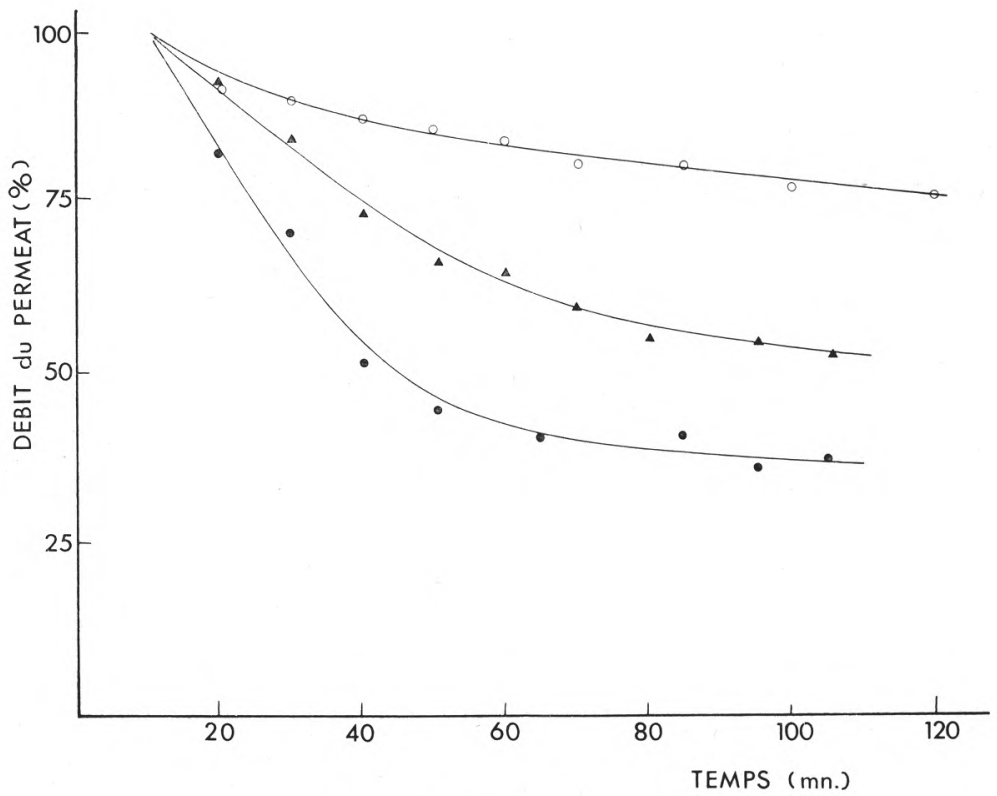

fig. 2

Influence de la température sur l'ultrafiltration d'ultrafiltrat de lait $\left(\mathrm{O}-\mathrm{O} 10^{\circ} \mathrm{C} ; \triangle 20^{\circ} \mathrm{C} ; \bullet-\bullet 30^{\circ} \mathrm{C}\right)$ 


\section{II. - Comportement de l'ultrafiltrat de lait dans le réacteur enzymatique}

On observe une diminution du débit du perméat dans les premières minutes d'ultrafiltration. Cette chute de débit est d'autant plus accentuée que la température est plus élevée ; ensuite le débit se maintient à un niveau constant et est plus important à $25^{\circ} \mathrm{C}$ qu'à $30^{\circ} \mathrm{C}$ (fig. 2).

Le chauffage de l'ultrafiltrat de lait à des températures supérieures à $30^{\circ} \mathrm{C}$ provoque l'apparition d'un précipité très fin au sein de ce liquide. Ce précipité qui se dépose très lentement est d'autant plus important que l'intensité du traitement thermique est plus forte. L'étude des teneurs en calcium de l'ultrafiltrat de lait mis en ouvre dans le réacteur et du perméat obtenu montre que la chute de débit observée à $30^{\circ} \mathrm{C}$ dans le réacteur est à attribuer au colmatage des membranes d'ultrafiltration par ce précipité de sels de calcium (tab. 4). Le passage d'une solution d'acide citrique (0,3 M) après rinçage du module à l'eau distillée, solubilise ce dépôt de

\section{TABLEAU 4}

Influence de la teneur en $\mathrm{Ca}$ de l'ultrafiltrat sur la rétention au niveau des membranes

\begin{tabular}{|c|c|c|}
\hline & $\begin{array}{r}\text { Concentration e } \\
\mathrm{d}^{\prime} \mathrm{u}\end{array}$ & $\begin{array}{l}\text { n) après } 20 \mathrm{mn} \\
\text { on }\end{array}$ \\
\hline $\begin{array}{c}\text { Concentration en Ca (ppm) de l'U.F. } \\
\text { de départ }\end{array}$ & Ultrafiltrat & Perméat \\
\hline 350 & 350 & 320 \\
\hline 290 & 290 & 290 \\
\hline
\end{tabular}

sels de calcium (fig. 3). Ce phénomène de colmatage des membranes par les sels de calcium présents dans l'ultrafiltrat de lait limitant grandement les performances du réacteur à membrane, nous avons recherché un moyen permettant d'éliminer les sels précipitables ou bien de les conserver sous forme soluble.

L'élimination des sels de calcium précipitables de l'ultrafiltrat de lait peut être réalisée en provoquant le dépôt de ces sels sur 


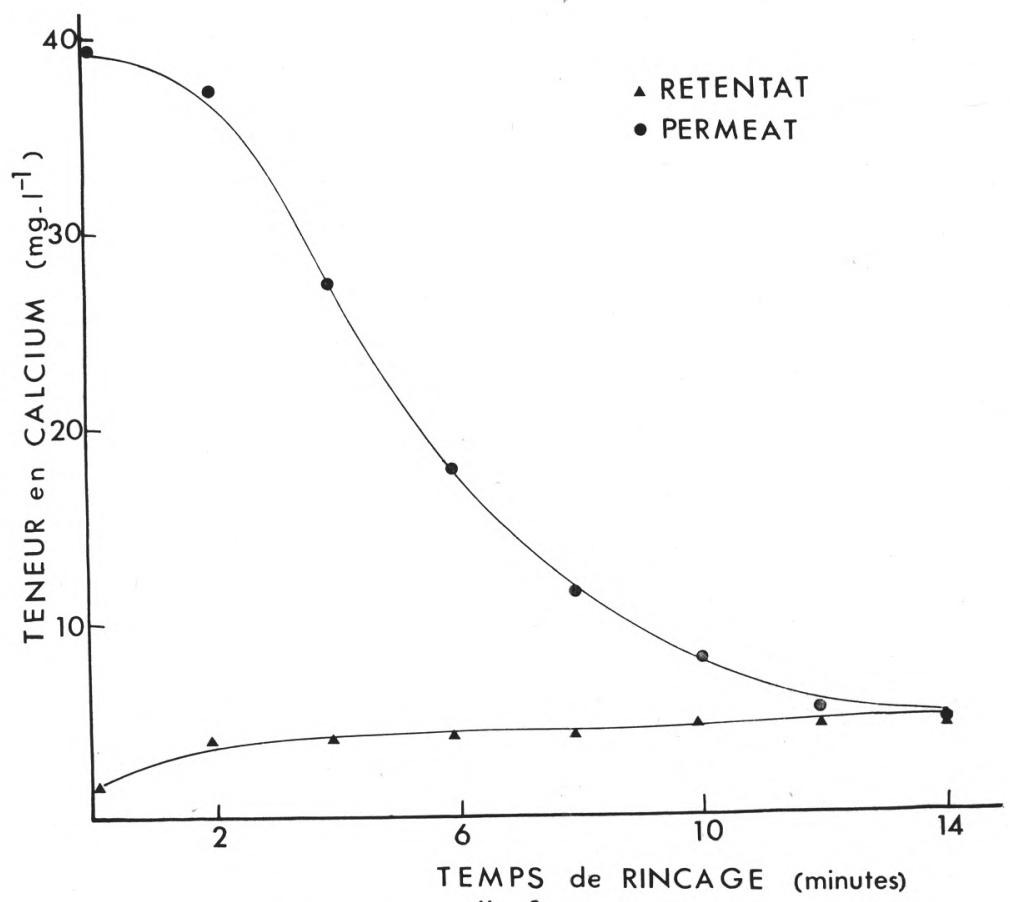

fig. 3

Rinçage du module après ultrafiltration, par une solution d'acide citrique $0,3 \mathrm{M}$

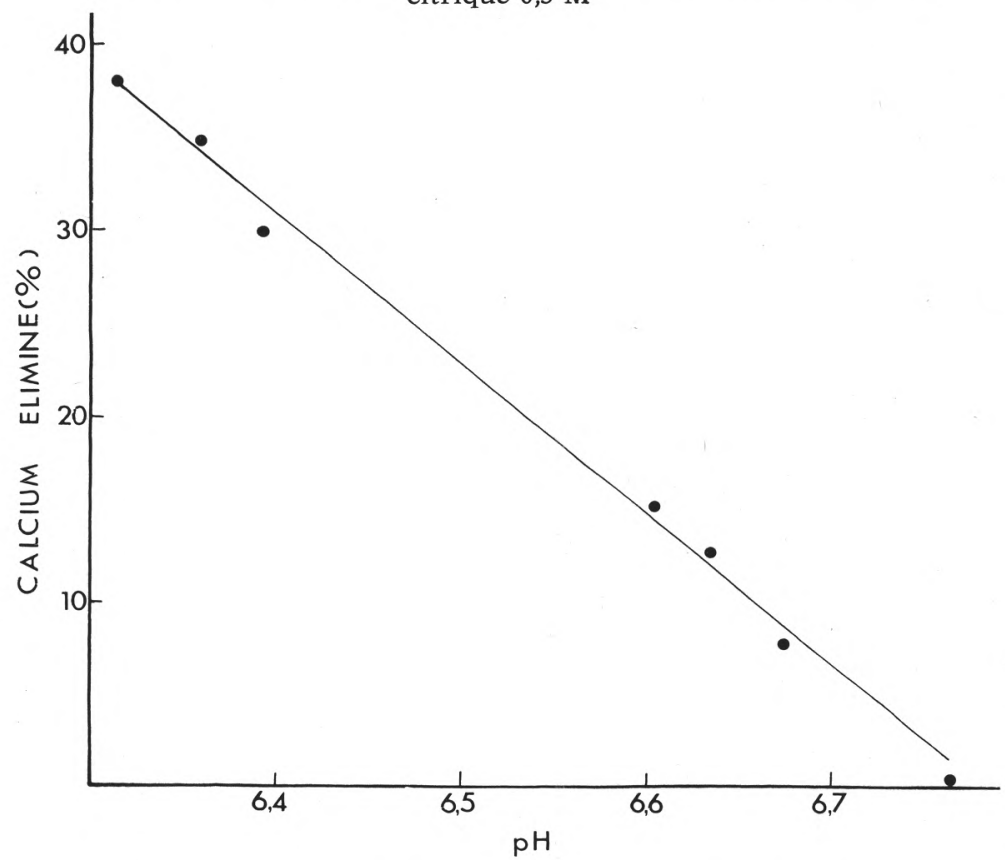

fig. 4

Influence de l'élimination du calcium de l'ultrafiltrat par chauffage sur son $\mathrm{pH}$ 


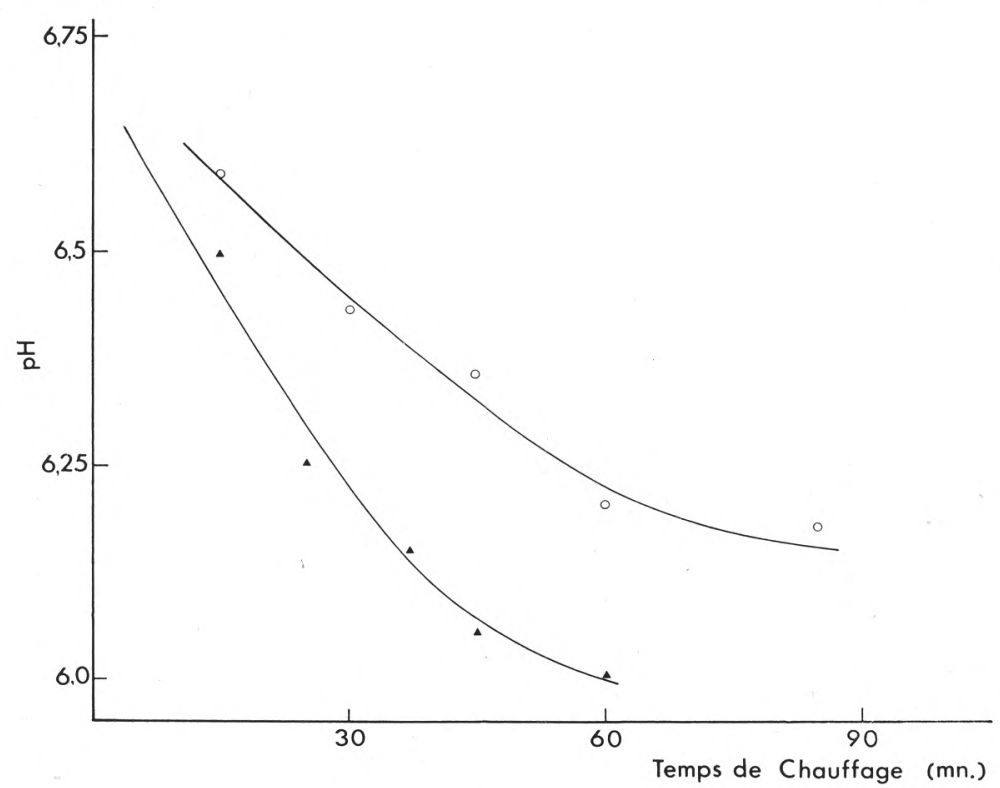

fig. 5

Précipitation du calcium de l'ultrafiltrat par chauffage

$\left(0-070^{\circ} \mathrm{C} ; \Delta-\Delta 85^{\circ} \mathrm{C}\right)$

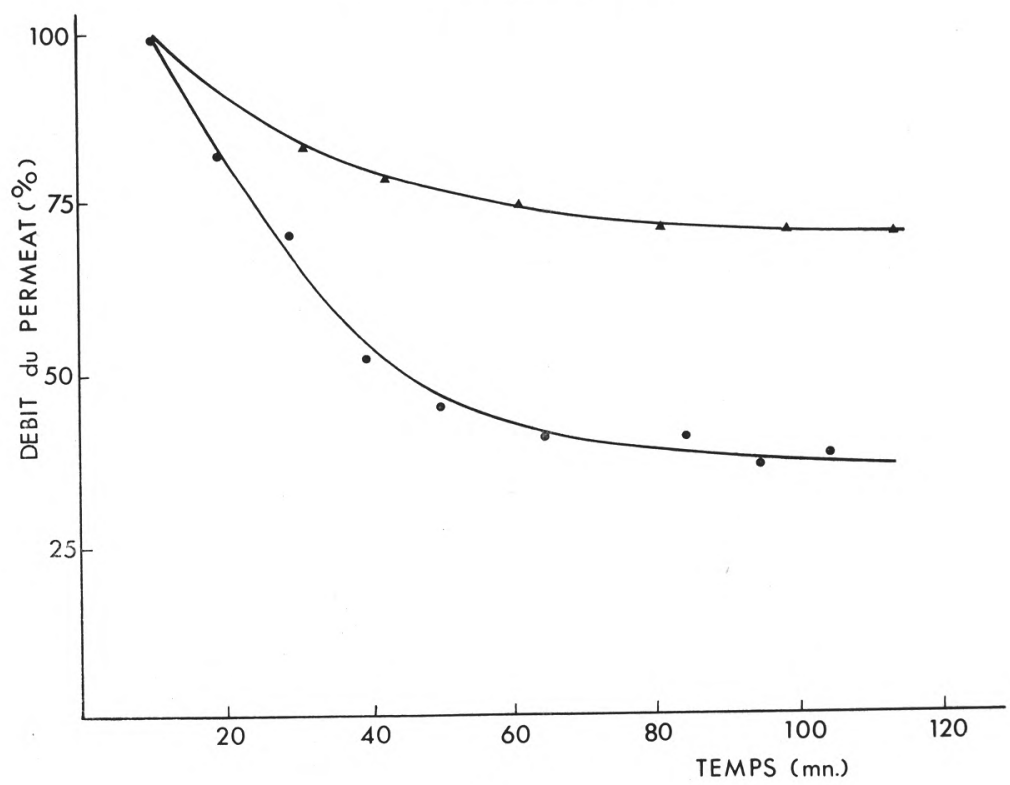

fig. 6

Influence de l'élimination du calcium, par chauffage, sur le débit de perméat, lors de l'ultrafiltration d'ultrafiltrat $\left(30^{\circ} \mathrm{C}\right)$ (- $\bullet$ témoin ; $₫$ - Ca éliminé 15 p. 100) 
une surface métallique chauffante (pasteurisateur par exemple). Une telle précipitation entraîne une diminution du $\mathrm{pH}$ de l'ultrafiltrat de lait, et nous avons montré qu'il existait une relation linéaire entre la valeur de ce $\mathrm{pH}$ et la quantité de $\mathrm{Ca}$ éliminé (fig. 4). La mesure du $\mathrm{pH}$ de l'ultrafiltrat de lait après traitement thermique peut ainsi constituer une bonne approximation de la quantité de calcium précipité. La figure 5 rassemble les résultats obtenus à $70^{\circ} \mathrm{C}$ et à $85^{\circ} \mathrm{C}$ pour des temps de chambrage allant juscu'à $90 \mathrm{mn}$. Pour chacune des deux températures étudiées, un palier de précipitation maximale existe à partir d'une certaine valeur du couple tempstempérature.

L'alcalinisation à température ambiante de l'ultrafiltrat de lait entraîne également la précipitation des sels de calcium. L'effet du traitement thermique sur les sels de calcium de l'ultrafiltrat de lait est accentué si au préalable, ce liquide est alcalinisé. Ainsi, l'ajustement à $\mathrm{pH} \mathrm{7,2} \mathrm{d'un} \mathrm{ultrafiltrat} \mathrm{de} \mathrm{lait}(\mathrm{pH}$ original $=6,5)$ par addition de $\mathrm{NaOH} \mathrm{N} / 10$, conduit à la précipitation de $45 \mathrm{p} .100 \mathrm{du}$ calcium après chauffage à $85^{\circ} \mathrm{C}$ pendant $5 \mathrm{mn}$, alors que ce taux n'est atteint avec l'ultrafiltrat témoin qu'après $30 \mathrm{mn}$.

L'élimination d'une partie du calcium de l'ultrafiltrat de lait sous forme de sels précipités soit par chauffage, soit par alcalinisation à température ambiante suivie d'une séparation du précipité par centrifugation $(1000 \times \mathrm{g}$ pendant $30 \mathrm{mn})$ améliore considérablement le débit du perméat. Les courbes de débit indiquées dans la figure 6 montrent que l'élimination par chauffage à $60^{\circ} \mathrm{C}$ pendant $60 \mathrm{mn}$ et centrifugation à $1000 \times \mathrm{g}$ pendant $30 \mathrm{mn}$ de $15 \mathrm{p} .100$ des sels de calcium contenus dans un ultrafiltrat de lait entraîne une amélioration très nette du débit de perméation des membranes composant le réacteur enzymatique.

L'addition de citrate trisodique à l'ultrafiltrat de lait en maintenant le calcium sous forme soluble évite la formation du précipité calcique au cours du chauffage. Des essais systématiques ont montré qu'à partir d'une concentration en citrate trisodique supérieure à 0,15 g p. 100 aucune précipitation du calcium ne se

\section{TABLEAU 5}

Influence de l'addition de citrate trisodique sur le $\mathrm{pH}$ et la concentration en $\mathrm{Na}$ de l'ultrafiltrat

\begin{tabular}{l|c|c|c|c|c}
\hline Teneur de l'U.F. en citrate de Na (p 100) & 0 & 0,15 & 0,20 & 0,25 & 0,30 \\
\hline $\mathrm{pH}$ & 6,73 & 6,78 & 6,78 & 6,79 & 6,80 \\
\hline Teneur de l'U.F. en Na (ppm) & 500 & & & 1000 & \\
\hline
\end{tabular}


produisait dans l'ultrafiltrat de lait porté à des températures comprises entre $25^{\circ} \mathrm{C}$ et $85^{\circ} \mathrm{C}$.

L'addition de citrate trisodique déplace légèrement le $\mathrm{pH}$ de l'ultrafiltrat de lait vers les $\mathrm{pH}$ alcalins (tab. 5) et augmente sa teneur en sodium. Cet accroissement de la teneur en ions $\mathrm{Na}^{+}$a pour conséquence une diminution de l'activité de la lactase (taux d'hydrolyse inférieur à 15 p. 100 au bout de 30 mn), (Hill et Huber, 1971).

Pour la suite de notre étude, nous avons retenu cette deuxième solution. Le citrate trisodique était ajouté à la teneur de 0,25 p. 100 .

\section{III. - Hydrolyse en continu du lactose de l'ultrafiltrat de lait dans le réacteur enzymatique}

La figure 7 indique la variation du pourcentage d'hydrolyse du lactose en fonction du temps (hydrolyse en continu). A une température et à un $\mathrm{pH}$ proches de l'optimum $\left(33^{\circ} \mathrm{C}, \mathrm{pH} 6,6\right)$ et avec une dose de lactase de $0,8 \mathrm{~g} / \mathrm{kg}$ d'ultrafiltrat, il a été possible avec ce réacteur enzymatique d'obtenir un taux d'hydrolyse de 80 p. 100, avec un débit minimum de $10 \mathrm{l} / \mathrm{h} / \mathrm{m}^{2}$.

Aucune chute importante de l'activité enzymatique n'a pu être observée au bout de $8 \mathrm{~h}$ de fonctionnement du réacteur (tab. 6) ni

\section{TABLEAU 6}

Hydrolyse en continu du lactose par la lactase en réacteur enzymatique

\begin{tabular}{c|c|c}
\hline $\begin{array}{c}\text { Durée de fonctionnement } \\
\text { du réacteur }(\mathrm{h})\end{array}$ & $\begin{array}{c}\text { Pourcentage d'hydrolyse } \\
\text { dans le prélèvement }\end{array}$ & Débit $\left(1 / \mathrm{m}^{2}\right)$ \\
\hline 2 & 89 & 10 \\
4 & 92 & 10 \\
8 & 80 & 10 \\
\hline
\end{tabular}

lors de la réutilisation de cette même solution de lactase conservée à l'état congelé $\left(-30^{\circ} \mathrm{C}\right)$ pendant plusieurs semaines. La diminution du pourcentage moyen d'hydrolyse obtenu après congélation ( 59 p. 100 au lieu de 77 p. 100 avant congélation) était due à une dilution de l'enzyme lors de sa réutilisation. 


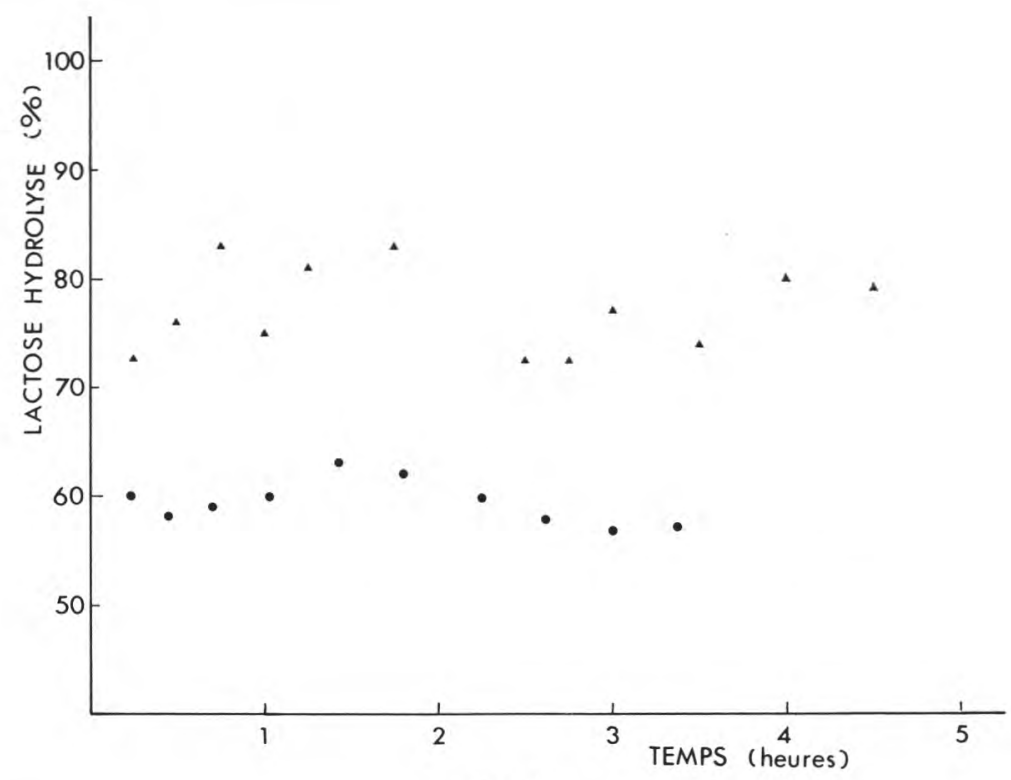

fig. 7

Hydrolyse en continu c.u lactose de l'ultrafiltrat en réacteur enzymatique (^ $\Delta 1^{\mathrm{e}}$ utilisation ; • réutilisation après congélation)

\section{IV. - Préparation d'un lait à faible teneur en lactose}

Le mélange d'un rétentat d'ultrafiltration de lait et d'ultrafiltrat à lactose partiellement hydrolysé permet la reconstitution d'un lait à faible teneur en lactose (fig. 8).

Le pourcentage d'hydrolyse à obtenir dans l'ultrafiltrat de lait pour s'assurer (à partir du mélange rétentat-ultrafiltrat) un taux de lactose donné dans le lait reconstitué, peut être calculé par la formule ci-après :

$$
\mathrm{X}=\frac{{ }^{\mathrm{F} Y}}{\mathrm{~F}-1} \quad \begin{aligned}
& \mathrm{X}=\text { p. } 100 \text { d'hydrolyse du lactose dans l'ultrafiltrat. } \\
& \mathrm{F}=\text { p. } 100 \text { d'hydrolyse du lactose dans le lait reconstitué. }
\end{aligned}
$$

La figure 9 permet, la valeur de $\mathrm{Y}$ étant choisie, de déterminer le couple $(\mathrm{X}, \mathrm{F})$ pour des valeurs de ces deux variables comprises respectivement entre 60 p. $100-90$ p. 100 et $2-6$.

Un exemple de fabrication de lait à faible teneur en lactose est donné dans le tableau 7.

Ce lait a été comparé à un lait à lactose non hydrolysé par un jury de dégustation (test de type tripartite ou encore appelé analyse 


\section{TABLEAU 7}

Composition des produits obtenus lors de la fabrication de lait reconstitué à lactose partiellement hydrolysé

\begin{tabular}{l|c|c|c|c}
\hline & $\begin{array}{c}\mathrm{EST} \\
(\mathrm{g} / \mathrm{kg})\end{array}$ & $\mathrm{pH}$ & $\begin{array}{c}\text { Facteur } \\
\text { de concen- } \\
\text { tration (F) }\end{array}$ & $\begin{array}{c}\text { Pourcen- } \\
\text { tage d'hy- } \\
\text { drolyse }\end{array}$ \\
\cline { 2 - 4 } & 10,8 & 6,70 & - & 0 \\
Lait & 27,7 & 6,70 & 4,10 & 0 \\
Rétentat & 5,3 & 6,75 & - & 0 \\
Ultrafiltrat & 5,3 & 6,75 & - & $76(\mathrm{X})$ \\
UF après passage dans le réacteur & 10,5 & 6,80 & - & $61(\mathrm{Y})$ \\
Lait reconstitué & & & & \\
\hline
\end{tabular}

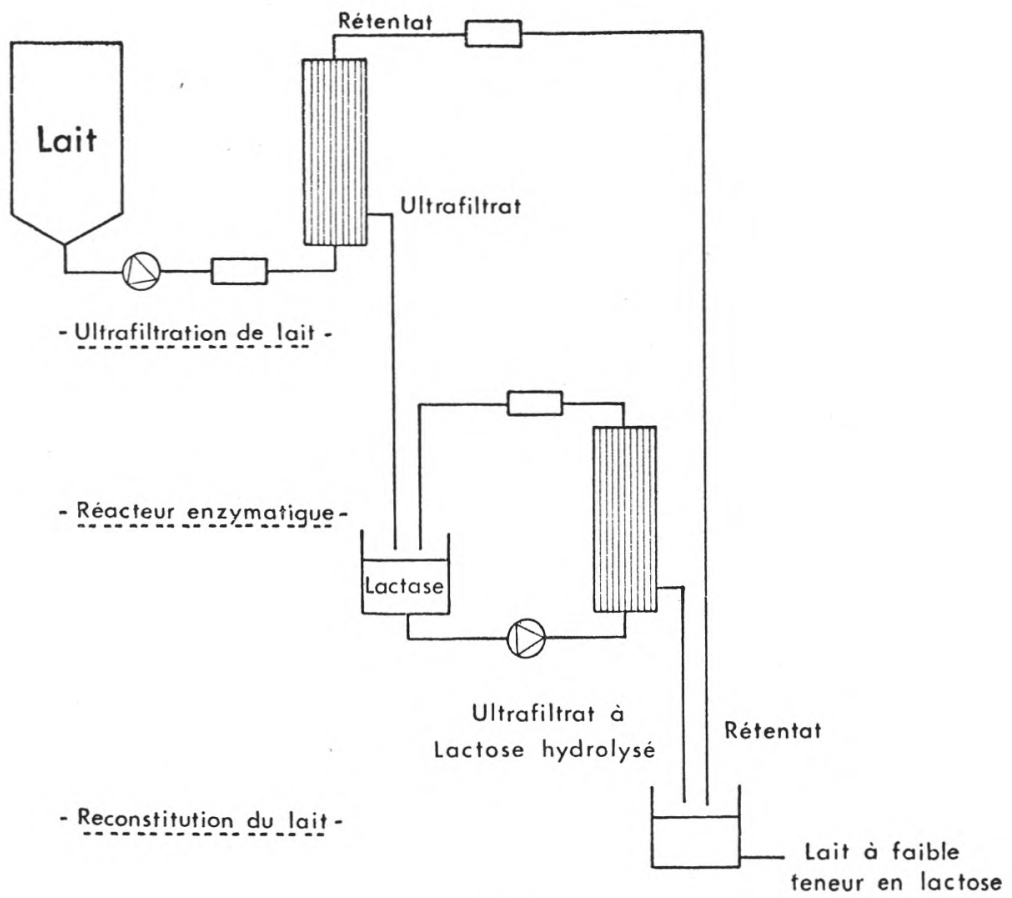

fig. 8

Diagramme de fabrication en coniinu de lait à faible teneur en lactose 


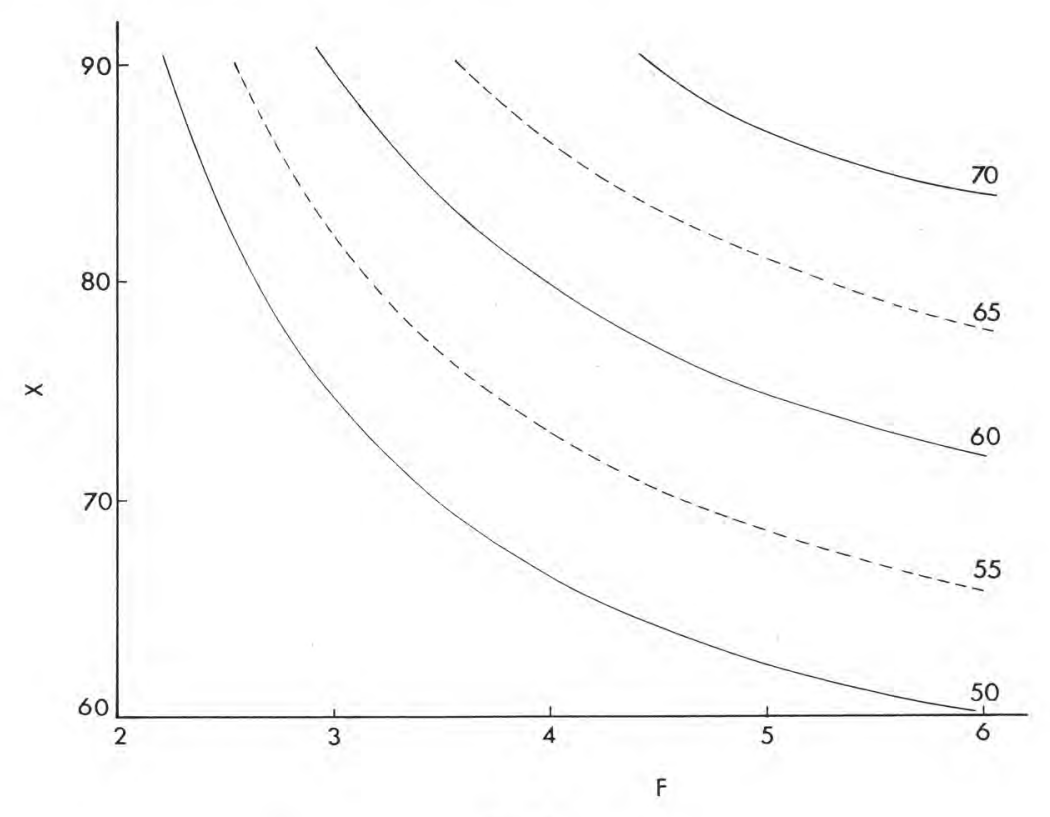

fig. 9

Pourcentage d'hydrolyse du lactose de l'ultrafiltrat en fonction du facteur de concentration F, pour différents pourcentages d'hydrolyse du lactose dans le lait.

de différence) avant et après pasteurisation afin de savoir si l'hydrolyse seule ou suivie d'un traitement thermique avait une influence sur le goût du lait. La réactivité du glucose et du galactose vis-à-vis des protéines au cours du chauffage est, en effet, plus élevée que celle du lactose (réaction de Maillard) (Olling, 1972).

Dans les deux tests, avant et après pasteurisation, on a observé une différence significative au seuil de 5 p. 100.60 p. 100 des dégustateurs étaient plutôt favorables au goût sucré du lait à lactose partiellement hydrolysé (le pouvoir sucrant du mélange glucose-galactose est, en effet, supérieur à concentration égale à celui du lactose).

La déminéralisation, préalable à l'hydrolyse, de l'ultrafiltrat de lait ou de lactosérum, modifie les conditions d'activité de la lactase. A la concentration en enzyme de $1 \mathrm{~g} / \mathrm{l}$, à une température de $34^{\circ} \mathrm{C}$ et au $\mathrm{pH}$ de 6,8 , seulement 47 p. 100 du lactose contenu dans l'ultrafiltrat de lactosérum déminéralisé étaient hydrolysés après $30 \mathrm{mn}$ contre 89 p. 100 dans l'ultrafiltrat de lait non déminéralisé.

Il est probable que cette différence d'activité soit à attribuer à la modification du rapport $\mathrm{K} / \mathrm{Na}$. Le potassium est en effet activateur 
de la réaction (Gould, 1975) alors que le sodium est considéré lui comme légèrement inhibiteur (Gist Brocades, 1974) :

- 4001 d'ultrafiltrat de lactosérum déminéralisé à 50 p. 100 ont été ainsi soumis à l'hydrolyse. Le produit obtenu, dont le lactose était hydrolysé à 85 p. 100 a été concentré par évaporation sous vide partiel jusqu'à une teneur en substance sèche de $81 \mathrm{~g} \mathrm{p} 100$ dans une installation Laguilharre. Translucide, légèrement coloré en jaune, le concentré obtenu présentait des caractéristiques rhéologiques voisines de celles du «miel».

\section{DISCUSSION}

L'hydrolyse enzymatique du lactose contenu dans l'ultrafiltrat de lait est réalisable, en continu, à un taux supérieur à 80 p. 100 en réacteur à membrane. La consommation d'enzyme avec la technologie proposée apparaît comme très faible, voire nulle puisqu'aucune perte d'activité de l'enzyme utilisée n'a pu être mise en évidence après $8 \mathrm{~h}$ de fonctionnement du réacteur.

Une faible élévation de la température de l'ultrafiltrat entraîne une précipitation des sels de calcium au niveau des membranes du réacteur. Il en résulte un colmatage des pores et une chute rapide du débit du perméat.

Ce phénomène ne s'observe pas lorsqu'on élève la température du lait soumis à ultrafiltration jusqu'à une valeur de $40^{\circ} \mathrm{C}-50^{\circ} \mathrm{C}$ (température limite des appareillages et des membranes actuellement commercialisés) et ce, bien que la quantité de calcium soluble diminue lorsqu'on soumet le lait à un traitement thermique (Pyne, 1958 ; Rose, 1959). Ceci conduit à supposer que les micelles de caséine, en complexant le calcium, limite la précipitation de ces sels au cours du chauffage. Mais le mécanisme de cette complexation reste à démontrer.

Les résultats obtenus par Hayes et al. (1974) et nos propres observations montrent que le comportement des sels minéraux dans le lactosérum serait identique à celui que nous observons dans l'ultrafiltrat de lait. Les chutes de débit de perméation observées lors du traitement du lactosérum par ultrafiltration seraient donc à attribuer non seulement au dépôt sur les membranes d'un film protéique mais aussi à un dépôt minéral plus ou moins important selon la nature du lactosérum mis en œuvre.

Le dépôt de sels de calcium sur les membranes d'ultrafiltration peut être évité :

— soit en déminéralisant partiellement l'ultrafiltrat de lait (ou de lactosérum) par traitement sur résines ou par électrodialyse ou 
bien par chauffage. Mais ces solutions ont pour inconvénients d'être coûteuses en énergie et de modifier la teneur en calcium du perméat à lactose hydrolysé obtenu et par suite celle du lait reconstitué,

- soit en formant un complexe soluble de citrate de calcium par addition de citrate trisodique. L'emploi de ce sel est autorisé dans l'industrie alimentaire (Dehove, 1971) lors de la fabrication de plusieurs produits (fromages fondus en particulier) mais l'augmentation de la teneur en sodium du produit final peut restreindre les utilisations notamment en diététique.

Néanmoins des études complémentaires devraient être entreprises en ce qui concerne:

a) les durées maximales de fonctionnement admissibles sur le plan bactériologique. Bien que les conditions de l'hydrolyse du lactose ( $\mathrm{pH}$ voisin de la neutralité - température voisine de $30^{\circ} \mathrm{C}$ ) soient favorables au développement des micro-organismes, on peut espérer que la contamination initiale sera très faible grâce à une désinfection efficace des installations d'ultrafiltration (Mocquot et al., 1975 ; Kiviniemi, 1974). En outre on peut stériliser par filtration les solutions de lactase,

b) les conditions de congélation, de conservation prolongée et de réutilisation des solutions de lactase; en effet il semble comme l'ont montré nos essais préliminaires que la congélation rapide en absence de lactose, c'est-à-dire une fois effectuée l'hydrolyse complète de ce sucre donne les meilleurs résultats. Mais il va de soi que ces opérations de congélation, conservation, décongélation devraient être répétées de très nombreuses fois.

L'hydrolyse du lactose en réacteur enzymatique peut permettre outre la fabrication en continu de lait ou de produits laitiers à lactose hydrolysé, la valorisation des ultrafiltrats de lait ou de lactosérum, sous-produits obtenus lors de la transformation du lait en fromage (Maubois et Mocquot, 1971) ou lors de la préparation de protéines purifiées de lactosérum. Après hydrolyse du lactose, ces ultrafiltrats peuvent être concentrés sans difficultés jusqu'à une teneur en matière sèche dépassant 80 p. 100. De tels concentrés à pouvoir sucrant intermédiaire entre ceux du lactose et du saccharose devraient présenter de l'intérêt pour d'autres industries agro-alimentaires (confiserie, confiturerie, biscuiterie).

La déminéralisation des ultrafiltrats à lactose hydrolysé destinés à de tels usages apparaît comme nécessaire en raison de la teneur relativement élevée en sels minéraux de ces produits ( 7 à 8 p. 100 de la matière sèche) et du goût salé qui en résulte aux fortes concentrations. La valorisation de tels concentrés de substitution devra donc non seulement prendre en compte le coût de l'hydrolyse du lactose mais aussi celui de la déminéralisation de l'ultrafiltrat. 


\section{Rés u m é}

La technique du réacteur enzymatique à membrane était appliquée à l'obtention de lait à lactose hydrolysé. Ce procédé nécessitait la séparation préalable par ultrafiltration de la phase aqueuse du lait ou ultrafiltrat. L'enzyme, une $\beta$ galactosidase commerciale était maintenue en solution dans le circuit rétentat du réacteur.

Les conditions optimales de l'hydrolyse du lactose contenu dans l'ultrafiltrat de lait et de lactosérum étaient déterminées. Le comportement des ultrafiltrats dans le réacteur était étudié dans les conditions ainsi définies.

Un procédé de fabrication, en continu, de lait à lactose hydrolysé, ou de produit de substitution du saccharose est proposé.

\section{S u $\mathbf{m} \mathbf{m}$ a r y}

Milk and milk by-products with a low lactose content were obtained by the application of the enzymatic ruembrane reactor technique. A previous separation of the aqueous phase of milk or ultrafiltrate was necessary and realized by ultrafiltration. The enzyme a commercial $\beta$-galactosidase, was maintained in solution in the retentate part of the membrane reactor.

The optimum conditions of the lactose hydrolysis in milk and whey ultrafiltrates were determined. The behaviour of the aqueous phase of milk in membrane reactor, specially of mineral salts, was studied. Two possibilities were proposed to avoid a calcium-phosphate deposit on the surface of (and in) the reactor membranes. The first one is a previous elimination of the calcium-phosphate by heat treatment ; the second one consists maintaining the calcium in a soluble form by adding a small amount of an alcaline citrate.

A continuous process for the lactose hydrolysis of milk and demineralized whey ultrafiltrate was proposed. The organoleptic quality of low lactose milk, before and after heat treatment, was evaluated by a tasting panel.

\section{Remerciements}

Nous remercions la firme Gist Brocades qui nous a fourni gracieusement la ß-galactosidase utilisée, M. Fauquant qui nous a apporté sa collaboration technique, $M$. Mocquot pour ses précieux conseils et $M$. Hermier pour ses suggestions et critiques dans la présentation de ce travail. 


\section{Bibliographie}

Davis (J. C.) (1974). - Kinetics studies in a continuous steady state hollow-fiber membrane enzyme reactor Biotech. and Bioeng., 16, 8, 1112-1122.

DeHove (R.A.) (1971). - La réglementation des produits alimentaires et non alimentaires. Commerce-éditions.

DE WAARD (H.) (1975). - Nutritional implications of lactose intolerance. News in Nutrition F.I.L. F. Doc.

Fallick (G. G.) (1969), - Industrial ultrafiltration. Process Biochemistry, 4, 9, 29-34.

Giacin (J. R.), Jakubowski (J.), Leeder (J. G.), Gilbert (S. G.), Kleyn (D. H.) (1974). Characterization of lactase immobilized on collagen : conversion of whey lactose by soluble and immobilized lactase. J. of Food Sc., 39, 4, 751-754.

Gist-BRocades nv industrial products division Delft-Holland. Technical data sheet.

Gould (B. J.) (1975). - Enzyme Data, Handbook of Enzyme Biotechnology. Halsted Press, John Wiley \& Son Inc. New York, Part II, Chap. 2, p. 142-143.

Hayes (J. F.), Dunkerley (J. A.), Muller (L. L.), Griffin (A. T.) (1974), - Studies on whey processing by ultrafiltration. II. Improving permeation rates by preventing fouling.The Australian J. of Dairy Tech., 37, 3, 132-140.

Hill (J. A.), Huber (R. E.) (1971). - Effects of various concentrations of $\mathrm{Na}^{+}$ and $\mathrm{Mg}^{++}$on the activity of $\beta$-galactosidase. Biochem. Biophys. Acta, 250-530.

Holzel (A.), Mereu (T.), Thompson (M. L.) (1962). - Severe lactose intolerance in enfancy. Lancet, 2, 1346.

Hyde (K. A.), Rothwell (J.) (1973). - Ice cream. Quality of ice cream : defects in ice cream. Chap. XI, 189-190. The Churchill Livingston, Edinburg and London.

Jakubowski (J.), Giacin (J. R.), Klein (D. H.), Gilbert (S. G.), Leeder (J. G.) (1975). - Effect of $\mathrm{Ca}, \mathrm{Mg}$ and whey proteins on the activity of betagalactosidase (A. niger) immobilized on collagen. J. of Food Sc., 40, 3, 467-469.

Jouany (J. P.) (1972). - Chromatographie en phase gazeuse des oses, des di et triholosides dans les milieux complexes. Ann. Biol. anim. Bioph. Biophys., 12, 3, 493-504.

Kiviniemi (L.) (1974). - Microbial growth during the ultrafiltration of sweet whey and skim milk. Kemia-Kemi, 12, 791-795.

KosikowsKi (F. V.), WIERZBICKI (L. E.) (1973). - Lactose hydrolysis in pasteurized milk by Saccharomyces lactis lactase. J. of Dairy Sc., 56, 1, 146-148.

Kosıкошsкi (F. V.) (1975). - Potential of enzymes in continous cheesemaking. J. Dairy Sci, 58, 7, 994-1000.

KRETCHMER (1971). - Gastroenterology, 61, 805, cité dans News in Nutrition F.I.L. F. Doc., 45, 1975.

Maubois (J. L.), Mocouot (G.) (1971). - Préparation de fromage à partir de " préfromage liquide " obtenu par ultrafiltration du lait. Le Lait, 51, 508, 495-533.

Mocouot (G.), Chopin (A.), Mahaut (M.), Maubots (J. L.) (1975). - Résultats non publiés.

Murthy (G. K.), RHEA (V.) (1967). - Determination of major cations in milk by atomic absorption spectrophotometry. J. Dairy Science, 50, 313-317.

Olling (C. J.) (1972). - Lactase treatment in dairy industry. Ann. Technol. Agric., $21,3,343-356$. 
Olson (A. C.), Cooney (C. L.) (1974). - Immobilized enzymes in food and microbial processes. Plenum Press, New York and London.

Pyne (G. T.) (1958). - The heat coagulation of milk. II. Variation in sensitivity of caseins to calcium ions. J. of Dairy Research, 25, 467-474.

Rose (D.), Tessier (H.) (1959). - Composition of ultrafiltrates from milk heated at 80 to $230^{\circ} \mathrm{F}$, in relation to heat stability. $J$. of Dairy Science, 42, 6, 969-980.

Vorsilak (P.), Maccoy (B.), Merson (R. L.) (1975). - Enzyme immobilized in a membrane sandwich reactor. J. of Food Sc., 40, 2, 431-432.

Webb (B. H.), Johnson (A. H.), Alford (J. A.) (1974). - Fundamentals of Dairy Chemistry. Frozen Dairy Products. Chap. XIV, 907-908. The Avi Publishing Company Inc., Westport, Connecticut.

Webb (B. H.), Johnson (A. H.), Alford (J. A.) (1974). - Fundamentals of Dairy Chemistry. Nutritional and physiological effects of lactose. Chap. VI, 314-319. The Avi Publishing Company Inc., Westport, Connecticut.

ZABORSKY (D. R.) (1974). - Immobilized enzymes. CRC Press, Cleveland Ohio. 